\title{
Magnetic helicity of a flux rope in the magnetotail: THEMIS results
}

\author{
Y. C. Zhang ${ }^{1}$, C. Shen ${ }^{1}$, Z. X. Liu ${ }^{1}$, and Y. Narita ${ }^{2}$ \\ ${ }^{1}$ State Key Laboratory of Space Weather, Center for Space Science and Applied Research, Chinese Academy of Sciences, \\ Beijing 100190, China \\ ${ }^{2}$ Institut für Geophysik und extraterrestrische Physik, Technische Universität Braunschweig, Mendelssohnstr. 3, \\ 38106 Braunschweig, Germany
}

Received: 19 May 2010 - Revised: 5 August 2010 - Accepted: 28 August 2010 - Published: 20 September 2010

\begin{abstract}
The magnetic field in many regions of magnetosphere has a complex topological structure. As a parameter to measure the topological complexity, the concept of magnetic helicity is a useful tool in magnetospheric physics. Here we present a case study of magnetic helicity in the flux rope (FR) in the near-Earth plasma sheet (PS) based on the in-situ observation from THEMIS for the first time. With the help of the Grad-Shafranov reconstruction technique, we determine the spatial distribution of magnetic field and evaluate the magnetic helicity in the flux rope. The conservation of magnetic helicity during multiple X-line reconnections and the transport of magnetic helicity between different magnetic field configurations are also discussed. The further application of helicity in magnetosphere will provide us more knowledge about the topologic property of the magnetic fields there and more attention should be paid to that.
\end{abstract}

Keywords. Magnetospheric physics (Magnetospheric configuration and dynamics; Plasma sheet) - Space plasma physics (Magnetic reconnection)

\section{Introduction}

Helicity integral (Moffatt, 1969) as a parameter to measure the complexity of the topology of a curve has been used to study the structures of vortex line, streamline and DNA (Pohl, 1980). When applied to magnetic field lines, it is called the magnetic helicity and measures twist, braid, shear or writhe of field lines. Magnetic helicity over a volume $V$ is defined as $H=\int_{V} \boldsymbol{A} \cdot \boldsymbol{B} \mathrm{d} V$ (Berger and Field, 1984), where the magnetic field is $\boldsymbol{B}=\nabla \times \boldsymbol{A}$ and $\boldsymbol{A}$ is the vector potential.

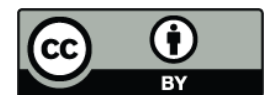

Correspondence to: Y. C. Zhang (zyc@cssar.ac.cn)
It is gauge invariant when the boundary of $V$ is a magnetic surface $\left(\left.B_{n}\right|_{s}=0\right.$ at the boundary $S$ of volume $V$ ), but in reality it is difficult to establish the magnetic surface. To guarantee the gauge invariance, Finn and Antosen (1985) defined the relative helicity for an open region,

$H_{\mathrm{r}}=\int_{V}\left(\boldsymbol{A}+\boldsymbol{A}^{\prime}\right) \cdot\left(\boldsymbol{B}-\boldsymbol{B}^{\prime}\right) \mathrm{d} V$,

where the reference magnetic field $\boldsymbol{B}^{\prime}\left(=\nabla \times \boldsymbol{A}^{\prime}\right)$ must satisfy the condition:

$$
\left.B_{n}\right|_{s}=\left.B_{n}^{\prime}\right|_{s} \text {. }
$$

The study of magnetic helicity has a history of half century and has been broadly applied to magnetic fields in space: remote sensing observation and simulation were performed for the solar active region (Pevtsov et al., 2003), while modeling and in-situ observations have been performed for magnetic clouds in the interplanetary space (Dasso et al., 2003; Hu and Dasgupta, 2005). Nevertheless the concept of magnetic helicity is less familiar in magnetosphere physics (Wright and Berger, 1989; Song and Lysak, 1989).

Under the interaction with solar wind, the magnetic field of the Earth is distorted from a normal dipolar field to form the magnetosphere (Dungey, 1961). Magnetic field lines in magnetotail become flattened, and especially those in the plasma sheet lean to the equator and are sheared (Shen et al., 2007, 2008). Additionally, due to magnetic reconnection, the magnetic field lines in the transition layers such as the magnetopause and the plasma sheet change their configuration sharply. Meso-small scale structures with a complex topology such as plasmoids and magnetic flux ropes are produced there (Russel and Elphic, 1979; Slavin et al., 2003). Many interesting magnetospheric processes (substorm, bursty bulk flows, etc.) are closely related to the configuration changes of magnetic field. Probably not every field line in the magnetosphere is smoothly connected between the Northern and the

Published by Copernicus Publications on behalf of the European Geosciences Union. 
Southern Hemisphere. The magnetic field lines with a complicated topology in many key regions of the magnetosphere serve the helicity study. However, the complexity of the magnetic field lines in these regions has been less investigated so far, and our works aim to apply the concept of magnetic helicity to the magnetosphere. Here we present a study of the magnetic helicity in a flux rope which is suited for the helicity study due to its twisted field line geometry. This work is based on the THEMIS-C spacecraft (THC) (Angelopoulos et al., 2008) performing in-situ observation in the near-Earth plasma sheet.

\section{Method description}

In a cylindrical magnetic flux rope, helical fields twist around its invariant axis $\hat{z}$. By cutting the flux rope with two planes (distance between them is $L$ along the axis) perpendicular to the axis, one obtains a volume $V$ composed of the side surface of the flux rope $\left(\left.B_{n}\right|_{\text {side }}=0\right)$ and two bottom surfaces $\left(\left.B_{n}\right|_{\text {bottom }}=\left.B_{\mathrm{Z}}\right|_{\text {bottom }}\right)$. If the reference field is chosen as $\boldsymbol{B}^{\prime}=B_{z} \hat{z}$, Eq. (2) is satisfied at two magnetically open bottoms and the relative helicity in the volume $V$ can be calculated uniquely. To get the relative helicity, the spatial distribution of the magnetic field $\boldsymbol{B}$ and the reference vector potential $\boldsymbol{A}^{\prime}$ corresponding to $\boldsymbol{B}^{\prime}$ must be calculated. A robust method to solve the distribution of $\boldsymbol{B}$ or $A_{\mathrm{z}}$ ( $z$ component of $\boldsymbol{A}$ ) on the cross-sectional plane x-y perpendicular to the axis $\hat{z}$ is the Grad-Shafranov (G-S) reconstruction technique. The GS reconstruction method (Hau and Sonnerup, 1999) is based on the following three assumptions: (1) The object to be reconstructed should have approximately a 2.5 dimension structure. (2) A frame must exist, in which the object to be studied is approximately temporally stationary. (3) In the frame, the inertial effect of the plasma can be neglected. If the plasma velocity in the frame is much smaller than the Alfvén speed, this assumption can be satisfied. The detailed description of this method and widespread applications can be found in Hau and Sonnerup (1999), Sonnerup et al. (2004), and Hasegawa et al. (2005). The most crucial issue in GS method is the determination of the invariant axis $\hat{z}$ which is clearly described by $\mathrm{Hu}$ and Sonnerup (2002). On the assumption of two-dimensional (2-D) MHD, the distribution of $\boldsymbol{B}$ or $A_{\mathrm{z}}$ can be obtained by solving the following G-S equation reduced from the momentum equation:

$\partial^{2} A_{\mathrm{z}} / \partial x^{2}+\partial^{2} A_{\mathrm{z}} / \partial y^{2}=-\mu_{0} d\left(P+P_{B z}\right) / d A_{\mathrm{z}}$,

where $P$ is the thermal pressure of plasma and $P_{B Z}=$ $B_{\mathrm{Z}}^{2} / 2 \mu_{0}$ is the magnetic pressure for the $B_{\mathrm{Z}}$ component along the invariant-axis, as the total magnetic field is represented as $\boldsymbol{B}=\nabla \times A_{\mathrm{z}} \hat{z}+B_{\mathrm{z}}(A) \hat{z}=\left[\partial A_{\mathrm{z}} / \partial y,-\partial A_{\mathrm{z}} / \partial x, B_{\mathrm{z}}(A)\right]$. As $\boldsymbol{B}=\nabla \times \boldsymbol{A}$ and $\boldsymbol{B}^{\prime}=\nabla \times \boldsymbol{A}^{\prime}=B_{\mathrm{Z}} \hat{z}$, the magnetic potential can be expressed as $\boldsymbol{A}=A_{\mathrm{z}} \hat{z}+\boldsymbol{A}^{\prime}$ and Eq. (1) reduces to

$$
\begin{aligned}
H_{\mathrm{r}} & =\int_{V} 2\left(A_{\mathrm{x}}^{\prime} B_{\mathrm{x}}+A_{\mathrm{y}}^{\prime} B_{\mathrm{y}}\right) d V \\
& =\int_{V} 2\left(A_{\mathrm{x}}^{\prime} \partial A_{\mathrm{z}} / \partial y-A_{\mathrm{y}}^{\prime} \partial A_{\mathrm{z}} / \partial x\right) \mathrm{d} V,
\end{aligned}
$$

and then the relative helicity of the flux rope in unit axial length $H_{\mathrm{r}} / L=\int_{x y} 2\left(A_{\mathrm{x}}^{\prime} \partial A_{\mathrm{z}} / \partial y-A_{\mathrm{y}}^{\prime} \partial A_{\mathrm{z}} / \partial x\right) \mathrm{d} x d y$ can be computed by integration over the cross-section. After getting the distribution of $A_{\mathrm{z}}$ from G-S reconstruction, it is crucial in the magnetic helicity calculation how to get $A_{\mathrm{x}}^{\prime}$ and $A_{\mathrm{y}}^{\prime}$. The reference vector potential $\boldsymbol{A}^{\prime}$ is related to the reference field $B_{\mathrm{Z}}$ by

$\partial A_{\mathrm{y}}^{\prime} / \partial x-\partial A_{\mathrm{x}}^{\prime} / \partial y=B_{\mathrm{z}}(x, y)$.

Two ways which are addressed by Hu and Dasgupta (2005) are utilized to get $A_{\mathrm{x}}^{\prime}$ and $A_{\mathrm{y}}^{\prime}$ with our small improvement on 2-D fit. The first is simple and takes the 2-D polynomial fit to $B_{\mathrm{Z}}$ spatial distribution, i.e., $B_{\mathrm{Z}}(x, y)=\sum_{m, n} a_{m, n} x^{m} y^{n}$. For this case $m=n=3$ is the best choice from all kinds of the combination of the exponents $(m, n)$ between 1 and 10 . We take all $B_{\mathrm{Z}}$ data in the 2-D polynomial fit instead of only several samples in Hu and Dasgupta (2005), so our 2-D polynomial fitting results are close to the true $B_{\mathrm{Z}}$ spatial distribution more. Once getting the coefficients $a_{m, n}$, we construct the polynomial expression of $A_{\mathrm{x}}^{\prime}$ and $A_{\mathrm{y}}^{\prime}$ through Eq. (5). The second (first addressed by Chae, 2001) is more rational and can easily be implemented. When imposing the Coulomb gauge to the reference vector potential, i.e.,

$\partial A_{\mathrm{x}}^{\prime} / \partial x+\partial A_{\mathrm{y}}^{\prime} / \partial y=0$,

and performing the Fast Fourier Transform (FFT) to Eqs. (5) and (6), one obtains $A_{\mathrm{x}}^{\prime}=F T^{-1}\left[\frac{i k_{y} F T\left(B_{\mathrm{z}}\right)}{k_{x}^{2}+k_{y}^{2}}\right]$ and $A_{\mathrm{y}}^{\prime}=$ $F T^{-1}\left[-\frac{i k_{x} F T\left(B_{z}\right)}{k_{x}^{2}+k_{y}^{2}}\right]$, where $k_{x}$ and $k_{y}$ are the wave numbers and $i=\sqrt{-1}$.

\section{THEMIS observation}

On 5 February 2009 at 06:25 UT, THC spacecraft was in an elliptical equatorial orbit, with its apogee in the magnetotail located at $(-18.42,-4.257,-3.999) R_{\mathrm{E}}\left(R_{\mathrm{E}}\right.$ : Earth radius $)$ in GSM coordinates. The FGM (Auster et al., 2008) and ESA (McFadden et al., 2008) experiments on board THC respectively provide high resolution magnetic field data and particle data in the interval surrounding this time. We use the 3-s resolution FGM and ESA data for HT analysis and 0.25-s resolution FGM data for the reconstruction calculation. Figure 1 shows THC observations from 06:24 to 06:27 UT in GSM coordinates. From top to bottom, the following parameters are plotted: ion density $\left(N_{\mathrm{i}}\right)$ and electron density $\left(N_{\mathrm{e}}\right)$, 


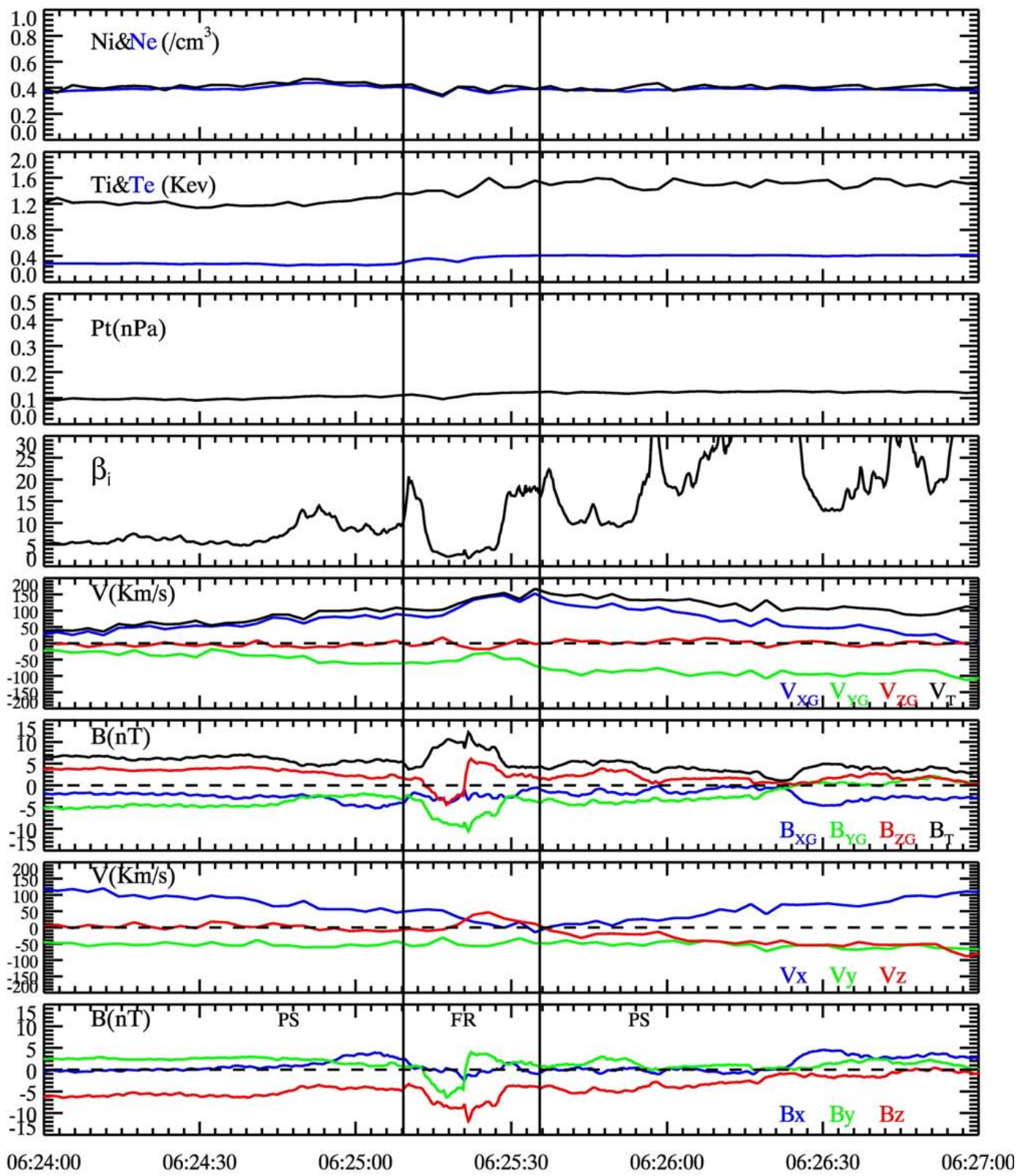

Fig. 1. THEMIS observation from 06:24 UT to 06:27 UT on 5 February 2009.

ion temperature $\left(T_{\mathrm{i}}\right)$ and electron temperature $\left(T_{\mathrm{e}}\right)$, plasma thermal pressure $\left(P_{\mathrm{t}}\right)$, plasma beta $\left(\beta_{i}\right)$, ion bulk velocity components $\left(V_{X G}, V_{Y G}, V_{Z G}\right)$ and bulk speed $\left(V_{\mathrm{T}}\right)$, magnetic field components $\left(B_{X G}, B_{Y G}, B_{Z G}\right)$ and total magnetic intensity $\left(B_{\mathrm{T}}\right)$, magnetic field components $\left(B_{\mathrm{X}}, B_{\mathrm{y}}, B_{\mathrm{Z}}\right)$ and ion bulk velocity components $\left(V_{\mathrm{x}}, V_{\mathrm{y}}, V_{\mathrm{z}}\right)$ in the local flux rope coordinates (i.e. $\mathrm{X}-, \mathrm{y}-, \mathrm{z}$-coordinates) . The implication of $B_{X G}<0$ places THC in the Southern Hemisphere and higher plasma beta values compared to the values in the lobe indicate that THC is in the southern plasma sheet at this time. Based on the $B_{Z G}$ signature, two vertical solid lines mark the boundary of the flux rope that will be used in G-S analysis.
As can be seen in the $Z G$ component of magnetic field (bipolar signal), at 06:25:09 UT THC enters the flux rope from the plasma sheet. $B_{Z G}$ decreases from $2.5 \mathrm{nT}$ to $-4 \mathrm{nT}$ and then increases to $7.1 \mathrm{nT}$. The peak-to-peak value of $B_{Z G}$ is $11.1 \mathrm{nT}$. Associated with this $B_{Z G}$ variation, there is a $\left|B_{Y G}\right|$ enhancement, increasing from $3 \mathrm{nT}$ to $10 \mathrm{nT}$. Furthermore, the $B_{Y G}$ enhancement leads to a peak in total magnetic field intensity, which is 2.2 times greater than the adjacent plasma sheet magnetic field. All these observations confirm the signals of flux rope as Zong et al. (2004) described. THC exits the flux rope at 06:25:36 UT and then $B_{Y G}$ and $B_{Z G}$ restore their original values in the plasma sheet. Due to the small 

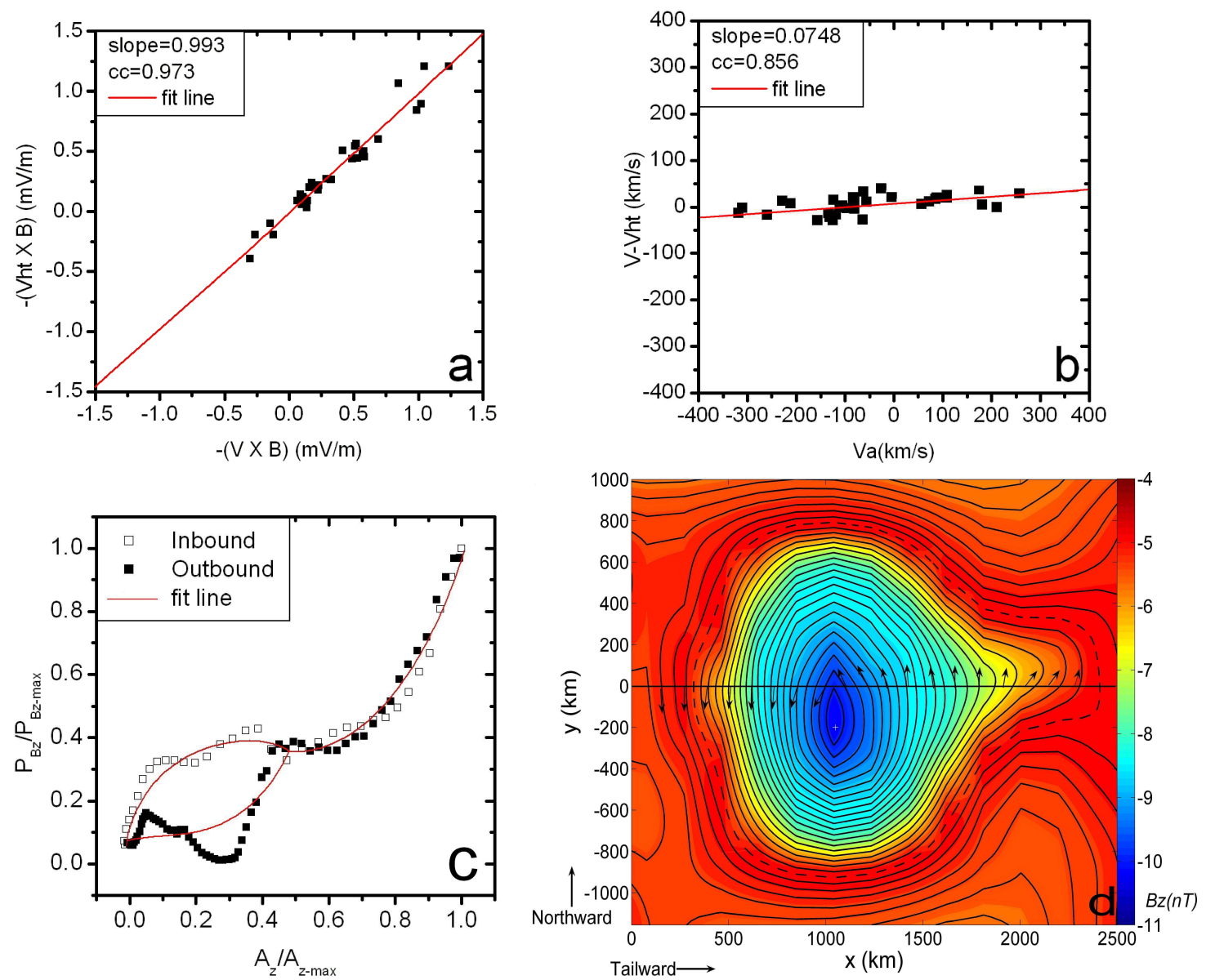

Fig. 2. (a) and (b) The result of deHoffman-Teller analysis. (c) Normalized magnetic pressure $P_{B z}$ versus normalized $A_{z}$. (d) The reconstruction results of the flux rope on 5 February 2009.

gradient of plasma thermal pressure $(\nabla P \approx 0)$, the flux rope will be reconstructed under the condition that only the contribution from the magnetic field is considered.

\section{Results and discussion}

A relatively good constant deHoffman-Teller (HT) frame velocity $(138.51,-63.85,47.34) \mathrm{km} / \mathrm{s}$ is found with the correlation coefficient between $-\left(\boldsymbol{V}_{\mathrm{HT}} \times \boldsymbol{B}\right)$ and $-(\boldsymbol{V} \times \boldsymbol{B})$ about 0.973 (Fig. 2a). In this HT frame the electric field vanishes $\left(\boldsymbol{E}=-\left(\boldsymbol{V}-\boldsymbol{V}_{\mathrm{HT}}\right) \times \boldsymbol{B} \approx 0\right)$ and the magnetic field is quasi-stationary $(\partial \boldsymbol{B} / \partial t=-\nabla \times \boldsymbol{E} \approx 0)$. The remanent velocity in the HT frame is almost negligible compared to the Alfvén velocity (Fig. 2b). All analysis imply the application of the G-S reconstruction technique to the magnetic field in this flux rope is appropriate. The direction of the invariant $(\hat{z})$-axis of the flux rope, the most important parameter in the reconstruction, is determined by searching for the direction which satisfies the following criteria: when THC moves inbound and outbound to the flux rope, the magnetic pressure $P_{B z}$ for the $B_{\mathrm{Z}}$ component along the proper invariant-axis should have the smallest difference for same $A_{\mathrm{z}}$ value (Hau and Sonnerup, 1999; Hu and Sonnerup, 2002), i.e., $\left[\sum_{m}\left(P_{B z \text {-inbound }}^{m}-P_{B z \text {-outbound }}^{m}\right)^{2}\right]^{\frac{1}{2}} /\left|\max \left(P_{B z}\right)-\min \left(P_{B z}\right)\right|$ has the minimum value. The most suitable invariant $(\hat{z})$-axis in the case here is mainly located in the dawn-dusk direction with the unit vector $(0.386,0.8976,-0.2126)$ in the GSM coordinates. The corresponding $\hat{x}$ and $\hat{y}$ separately direct to $(-0.9195,0.393,-0.1038)$ and $(0.0742,0.1995,0.9771)$. Figure $2 \mathrm{c}$ displays the plot of $P_{B z}$ variation versus $A_{z}$ (respectively normalized to their maxima) corresponding to the above invariant-axis. Red line in the figure is the fit lines from the polynomials and exponentials fitting. In the range $0.0-0.4$ of $A_{\mathrm{z}}, P_{B z}$ is obviously different between the inbound and the outbound orbits, which maybe reflects the different interaction of the flux rope at the leading edge and the trailing edge with the ambient plasmas as the flux rope moves toward the Earth with a higher speed. At the leading edge, the plasma ahead of the flux rope will compress the face of the flux rope, so $P_{B z}$ has the higher value than at the tail edge. At the trailing edge, the fast motion of the flux 

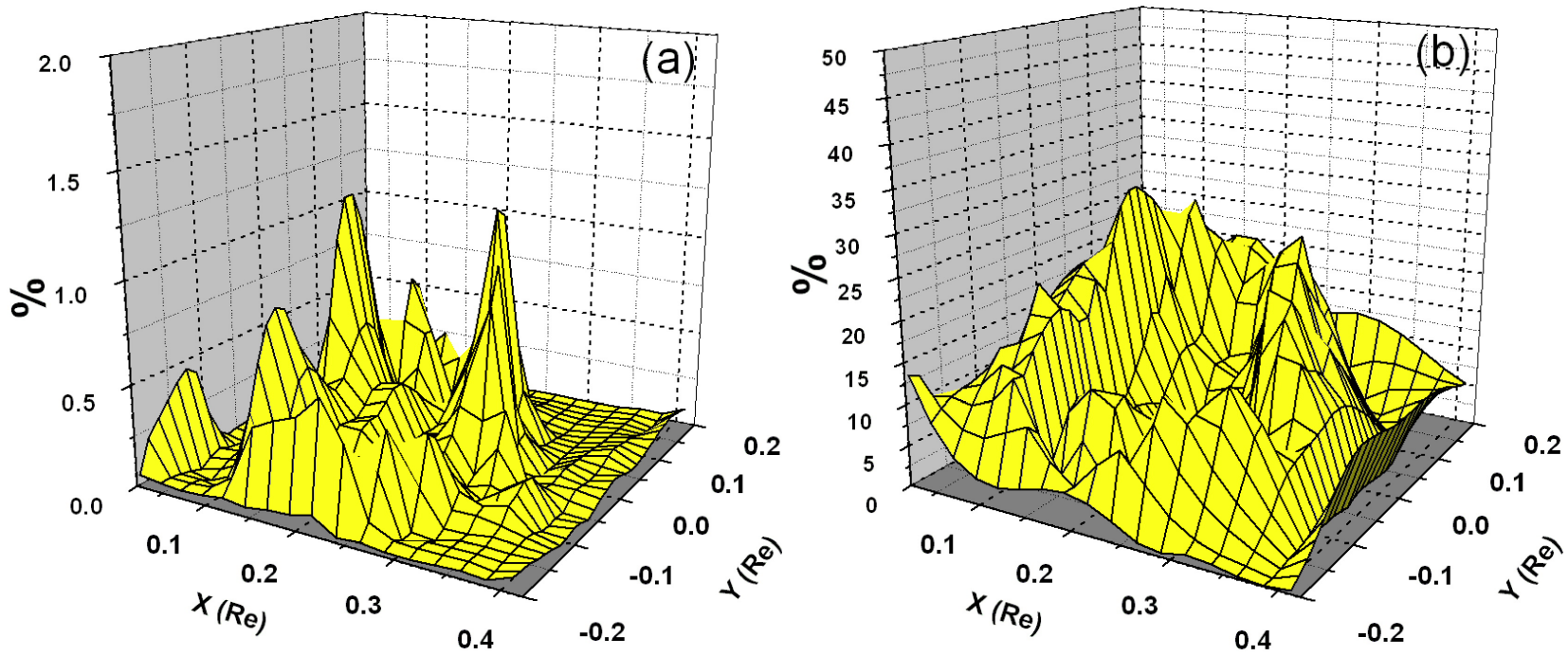

Fig. 3. The distribution of the discrepancy between the recovered $B_{\mathrm{Z}}$ and $B_{\mathrm{Z}}$ from G-S technique in the x-y plane: (a) for the recovered $B_{\mathrm{Z}}$ from FFT, (b) for the recovered $B_{\mathrm{Z}}$ from 2-D polynomial fit.

rope in the plasma sheet maybe produces the low pressure region behind the flux rope. Due to the trend of the plasma to the pressure equilibrium, $P_{B z}$ at the trailing part of the flux rope also has the lower value than at the leading part.

Figure $2 \mathrm{~d}$ presents the reconstructed distribution of $A_{\mathrm{z}}$ and $B_{\mathrm{Z}}$ in this case in the reconstruction coordinates $(\hat{x}, \hat{y}$ and $\hat{z}$ obtained above). The northern plasma sheet is at the top of the figure and the Earth is to the left. The closed black curves are the contour plots of the magnetic potential $A_{\mathrm{z}}$ in the cross-sectional x-y plane, which are just the magnetic field lines projected onto the cross section. The filled color inside the curves displays the distribution of the field component $B_{\mathrm{Z}}$ along the principal axis. The $B_{\mathrm{Z}}$ value for the different color is defined by the color bar at the right of the figure. The plus sign at the center of the figure denotes the strongest of the axial field, which is $B_{\mathrm{Z}}=-10.3 \mathrm{nT}$. The projection of the THC orbit on the cross section of the flux rope is the solid black line which also bears the arrows indicating the direction of the measured magnetic fields. In interpreting the G-S result, we must identify if other mechanisms such as the localized (3-D) bursty reconnection given guide field produce the signal of $B_{Z G}$ bipolar accompanied by $B_{Y G}$ enhancement in the original GSM coordinates (Shirataka et al., 2006). Hasegawa et al. (2007) reconstructed the synthetic data from a 3-D MHD reconnection simulation and their results show the great difference from the case of true flux rope. There are considerable perpendicular velocity components remnant in the HT frame and the resulted map is obviously elongated at $\mathrm{x}$-direction (see their Figs. 5 and 7). But in Fig. 2b and d, the negligible velocity remnant in the HT frame and the nearly round circles (except for the little outward protuberance at the tail side) help us to differentiate this flux rope from 3-D reconnection. Aim of the paper is to calculate the helicity in the flux rope, so the detail discussion about the structure of the flux rope in general can be seen at Shen et al. (2007) and Zhang et al. (2007).

Before calculating the helicity, we first check the accuracy of the $A_{\mathrm{x}}^{\prime}$ and $A_{\mathrm{y}}^{\prime}$ by evaluating the standard deviation $\sigma=\sqrt{\sum_{O}\left(B_{\mathrm{Z}, \text { recovered }}-B_{\mathrm{Z}, \mathrm{GS}}\right)^{2} / O}(O$ is the number of all $B_{\mathrm{Z}}$ ) between the recovered $B_{\mathrm{z}}$ with $A_{\mathrm{x}}^{\prime}, A_{\mathrm{y}}^{\prime}$ and $B_{\mathrm{z}}$ from G$\mathrm{S}$ technique. We take the $B_{\mathrm{Z}}$ from the G-S technique as the base and then compute the spatial distribution of discrepancy between the recovered $B_{\mathrm{z}}$ with $A_{\mathrm{x}}^{\prime}$ and $A_{\mathrm{y}}^{\prime}$ and the base (i.e. $\left.\left|B_{\mathrm{Z}, \text { recovered }}-B_{\mathrm{Z}, \mathrm{GS}}\right| / B_{\mathrm{Z}, \mathrm{GS}}\right)$ in the $\mathrm{x}-\mathrm{y}$ plane. Figure 3 shows this distribution in percentage: panel (a) displays discrepancy between $B_{\mathrm{Z}}$ from FFT and the base with $\sigma=0.09 \mathrm{nT}$, in which the maximum discrepancy is $1.2 \%$; while the discrepancy between $B_{\mathrm{Z}}$ from 2-D polynomial fit and the base is much greater as shown in panel (b), in which the maximum discrepancy is $30 \%$ and $\sigma=0.8 \mathrm{nT}$. Obviously because of the smaller $\sigma$ and smaller discrepancy, the result from FFT is more reliable.

Relative helicity integrals over the region enclosed by dashed contour line $\left(B_{\mathrm{Z}}=-5.0 \mathrm{nT}\right)$ in Fig. $2 \mathrm{~d}$ give $H_{\mathrm{r}} /\left.L\right|_{\mathrm{FFT}}=-0.386 \mathrm{nT}^{2} R_{\mathrm{E}}^{3}$ with $A_{\mathrm{x}}^{\prime}$ and $A_{\mathrm{y}}^{\prime}$ from FFT and $H_{\mathrm{r}} /\left.L\right|_{2 \mathrm{D}-\text { fit }}=-0.421 \mathrm{nT}^{2} R_{\mathrm{E}}^{3}$ with $A_{\mathrm{x}}^{\prime}$ and $A_{\mathrm{y}}^{\prime}$ from 2-D polynomial fit, respectively. These values represent the extent to which the fields in the flux rope twist, while the negative sign implies that the twist of the fields around the axis of this flux rope follows the left-hand sense. Due to less previous works about magnetic helicity in magnetosphere, our result is not so intuitional. Hu and Dasgupta (2005) calculated the magnetic helicity density $\left(H_{\mathrm{r}} / V\right)$ in a magnetic cloud with same method and Narita et al. (2009) evaluated that in 
the Earth's foreshock region. The magnetic helicity density in this case is $-7.72 \mathrm{nT}^{2} R_{\mathrm{E}}$ and is not comparable to their results quantitatively. Although comparison between these different magnetic structures makes no more sense, the increasing investigation could improve our intuition about magnetic helicity.

Most interesting topic in magnetic helicity study is the helicity transport between different magnetic field regions and the helicity conservation during reconnection. As we know, the magnetic flux rope is the direct production of multiple $\mathrm{X}$ line reconnection process in the background magnetic fields (Lee, 1995; Slavin et al., 2003). But retrospecting the origin of the magnetic helicity in the plasma sheet flux rope, we must consider the role of the interplanetary magnetic fields (IMF). Cowley (1981) argued that IMF $B_{\text {y }}$ component can be mapped to the plasma sheet by the process such as magnetic reconnection at the magnetopause and shear the magnetic fields in the northern and southern plasma sheet. It is just the appearance of the y-components of magnetic fields in the plasma sheet that favor the production of the flux rope. So essentially, the helicity in the flux rope originates from the IMF-geomagnetic fields interaction which is the beginning of a series of changes in the magnetospheric fields. The helicity is transported during these changes and the helicity of the plasma sheet flux rope is an important phase of the cascade of magnetic helicity in the magnetospheric system. From the calculation of the helicity of the flux rope in the plasma sheet, we can partially learn the information about the helicity (topology characteristics) of the other magnetospheric fields, especially the plasma sheet fields. That work needs more observations than presented here, but we still have a discussion in theory. In the process of multiple X-line reconnection, the magnetic field relaxes the magnetic energy to the configuration with a minimum energy state (for example the force-free flux rope), often called the Taylor state (Taylor, 1974). Also Taylor (1986) and Berger and Field (1984) point that magnetic helicity is approximately conserved during magnetic reconnection though the configuration of magnetic field change. In the flux rope case here, the configuration changes from the sheared fields in the plasma sheet before reconnection to the helical fields in the flux rope after reconnection. The sheared fields in the plasma sheet bear the mutual-helicity, while the fields in flux rope bear selfhelicity. So the mutual-helicity of the sheared fields is transformed to the self-helicity in the flux rope in part and to the helicity beared by the other fields around the flux rope through multiple X-line reconnection. Due to the limitation of observation, what proportion the helicity in flux rope occupies in the total helicity in plasma sheet can not be determined. But this work has a forward step to the investigation of the magnetic helicity of the flux rope which is closely related to the configuration of the plasma sheet fields and multiple- $X$ line reconnection there. The multiple- $X$ line reconnection in the plasma sheet relaxes the magnetic energy and changes the magnetic connectivity, while this progress also can be seen as the helicity redistribution in the plasma sheet as discussed by Wright and Berger (1989). In aspects of understanding the complicated structure of the magnetic field, the magnetic helicity is as vigorous as other physical parameters such as energy and flux. Combined with more future observations, our results can give more information in investigating the complexity of the fields in the flux rope and the background fields.

Further application of this helicity calculation to the population of plasma sheet flux ropes will give us the previously unknown knowledge about the fields with different structure, i.e., force-free vs. highly non-force-free flux ropes. When the flux ropes move earthward or tailward, the effect of the helicity in them on near-Earth region or deeper space (Moon?) add more significance behind the single calculation of the helicity. Additionally the helicity analysis also serves as a diagnostic tool to the magnetic reconnection (Wiegelmann and Büchner, 2001).

Acknowledgements. This work was supported by the National Natural Science Foundation of China Grant No. 40804033, 40974101, 40674094 and 40731054, Ministry of Science and Technology of China Grant 2006CB806305, the Hundred Talents Program of the CAS, and Project Supported by the Specialized Research Fund for State Key Laboratories.

We acknowledge NASA contract NAS5-02099 and V. Angelopoulos for use of data from the THEMIS Mission. Specifically: K. H. Glassmeier, U. Auster and W. Baumjohann for the use of FGM data provided under the lead of the Technical University of Braunschweig and with financial support through the German Ministry for Economy and Technology and the German Center for Aviation and Space (DLR) under contract 50 OC 0302, and C. W. Carlson and J. P. McFadden for use of ESA data.

Topcial Editor R. Nakamura thanks J. A. Slavin and H. Zhang for their help in evaluating this paper.

\section{References}

Angelopoulos, V.: The THEMIS Mission, Space Sci. Rev., 141, 534, 2008.

Auster, H.-U., Glassmeier, K.-H., Magnes, W., Aydogar, O., Baumjohann, W., Constantinescu, D., Fischer, D., Fornacon, K.H., Georgescu, E., Harvey, P., Hillenmaier, O., Kroth, R., Ludlam, M., Narita, Y., Nakamura, R., Okrafka, K., Plaschke, F., Richter, I., Schwarzl, H., Stoll, B., Valavanoglou, A., and Wiedemann, M.: The THEMIS Fluxgate Magnetometer, Space Sci. Rev., 141, 235-264,2008.

Berger, M. A. and Field, G. B.: The topological properties of magnetic helicity, J. Fluid. Mech., 147, 133-148, 1984.

Chae, J.: Observational determination of the rate of magnetic helicity transport through the solar surface via the horizontal motion of fieldline footprints, Astrophys. J., 560, L95-L98, 2001.

Cowley, S. W. H.: Magnetospheric asymmetries associated with the Y component of the IMF, Planet Space Sci., 29, 79-96, 1981.

Dasso, S., Mandrini, C. H., Démoulin, P., and Farrugia, C. J.: Magnetic helicity analysis of an interplanetary twisted flux tube, J. Geophys. Res., 108(A10), 1362, doi:10.1029/2003JA009942, 2003. 
Dungey, J. M.: Interplanetary magnetic field and the aural zones, Phys. Rev. Lett., 6, 47-48, 1961.

Finn, J. M. and Antonsen, T. M.: Magnetic helicity: What is it and what it is good for?, Comments Plasma Phys. Control. Fusion., 9(3), 111-126,1985.

Hasegawa, H., Sonnerup, B. U. Ö., Klecker, B., Paschmann, G., Dunlop, M. W., and Rème, H.: Optimal reconstruction of magnetopause structures from Cluster data, Ann. Geophys., 23, 973982, doi:10.5194/angeo-23-973-2005, 2005.

Hasegawa, H., Nakamura, R., Fujimoto, M., Sergeev, V. A., Lucek, E. A., Réme, H., and Khotyaintsev, Y.: Reconstruction of a bipolar magnetic signature in an earthward jet in the tail: Flux rope or 3D guide-field reconnection?, J. Geophys. Res., 112, A11206, doi:10.1029/2007JA012492, 2007.

Hau, L.-N. and Sonnerup, B. U. Ö.: Two-dimensional coherent structures in the magnetopause: Recovery of static equilibria from single-spacecraft data, J. Geophys. Res., 104, 6899-6917, 1999.

$\mathrm{Hu}$, Q. and Dasgupta, B.: Calculation of magnetic helicity of cylindrical flux rope, Geophys. Res. Lett., 32, L12109, doi:10.1029/2005GL023004, 2005.

$\mathrm{Hu}$, Q. and Sonnerup, B. U. Ö.: Reconstruction of magnetic cloudsin the solar wind: Orientations and configurations, J. Geophys. Res., 107(A7), 1142, doi:10.1029/2001JA000293, 2002.

Lee, L. C.: A review of magnetic reconnection: MHD models, in physics of the magnetopause, in: Geophysical Monographs Series, edited by: Song, P., Sonnerup, B. U. Ö., and Thomsen, M. F., 90. Washington, D.C., AGU, 139, 1995.

McFadden, J. P., Carlson, C. W., Larson, D., Ludlam, M., Abiad, R., Elliott, B., Turin, P., Marckwordt, M., and Angelopoulos, V.: The THEMIS ESA plasma instrument and in-flight calibration, Space Sci. Rev., 141, 277-302, 2008.

Moffatt, H. K.: The degree of knottedness of tangled vortex lines, J. Fluid. Mech., 35, 117-129, 1969.

Narita, Y., Kleindienst, G., and Glassmeier, K.-H.: Evaluation of magnetic helicity density in the wave number domain using multi-point measurements in space, Ann. Geophys., 27, 39673976, doi:10.5194/angeo-27-3967-2009, 2009.

Pevtsov, A. A., Maleev, V. M., and Longcope, D. W.: Helicity Evolution in Emerging Active Regions, Astrophys. J., 593, 12171225, 2003.

Pohl, W. F.: DNA and differential eometry, Math.Intelligencer, 3, 20-27, 1980.

Russel, C. T. and Elphic, R. C.: ISEE observations of flux transfer events at the dayside magnetopause, Geophys. Res. Lett., 6, 33$36,1979$.
Shen, C., Li, X., Dunlop, M., Shi, Q. Q., Liu, Z. X., Lucek, E., and Chen, Z. Q.: Magnetic Field Rotation Analysis and the Applications, J. Geophys. Res., 112(A6), A06211, doi:10.1029/2005JA011584, 2007.

Shen, C., Liu, Z., Li, X., Dunlop, M. W., Lucek, E. A., Rong, Z., Chen, Z., Escoubet, C. P., Malova, H. V., Lui, A. T. Y. , Fazakerley, A. N., Walsh, A. P., and Mouikis, C.: Flattened Current Sheet and its Evolution in Substorms, J. Geophys. Res., 113, A07S21, doi:10.1029/2007JA012812,2008.

Shirataka, N., Fujimoto M., Hasegawa H., and TanDokoro R.: Reproducing the bipolar magnetic signature at the jet leading edge by three-dimensional reconnection with nonzero guide field, J. Geophys. Res., 111, A07201, doi:10.1029/2005JA011521,2006.

Slavin, J. A., Lepping, R. P., Gjerloev, J., Fairfield, D. H., Hesse, M., Owen, C. J., Moldwin, M. B., Nagai, T., Ieda, A., and Mukai, T.: Geotail observations of magnetic flux ropes in the plasma sheet, J. Geophys. Res., 108(A1), 1015, doi:10.1029/2002JA009557, 2003.

Sonnerup, B. U.Ö., Hasegawa, H., and Paschmann, G.: Anatomy of a flux transfer event seen by Cluster, Geophys. Res. Lett., 31, L11803, doi:10.1029/2004GL020134, 2004.

Song, Y. and Lysak, R. L: Evaluation of twist helicity in flux transfer event tubes. J. Geophys. Res., 94(A5), 5273-5281, 1989.

Taylor, J. B.: Relaxation of Toroidal Plasma and Generation of Reverse Magnetic Fields, Phys. Rev. Lett., 33, 1139-1141, 1974.

Taylor, J. B.: Relaxation and magnetic reconnection in plasmas, Rev. Mod. Phys., 58, 741-763, 1986.

Wiegelmann, T. and Büchner, J.: Evolution of magnetic helicity in the course of kinetic magnetic reconnection, Nonlin. Processes Geophys., 8, 127-140, doi:10.5194/npg-8-127-2001, 2001.

Wright, A. N. and Berger, M. A.: The effect of reconnection upon the linkage and interior structure of magnetic flux tubes, J. Geophys. Res., 94(A2), 1295-1302, 1989.

Zhang, Y. C., Liu, Z. X., Shen, C., Fazakerley, A., Dunlop, M., Réme, H., Lucek, E., Walsh, A. P., and Yao, L.: The magnetic structure of an earthward-moving flux rope observed by Cluster in the near-tail, Ann. Geophys., 25, 1471-1476, doi:10.5194/angeo-25-1471-2007, 2007.

Zong, Q. G., Fritz T. A., Spence, H., Zhang, H., Huang, Z. Y., $\mathrm{Pu}$, Z. Y., Glassmeier, K.-H., Korth, A., Daly, P. W., Balogh, A., and Réme, H.: Cluster observations of earthward flowing plasmoid in the tail, Geophys. Res. Lett., 31, L18803, doi:10.1029/2004GL020692, 2004. 